\title{
ON THE SIGN DISTRIBUTIONS OF HILBERT SPACE FRAMES
}

\author{
NIKOLAI NIKOLSKI, ALEXANDER VOLBERG
}

\begin{abstract}
We show that the positive and negative parts $u_{k}^{ \pm}$of any frame in a real $L^{2}$ space with respect to a continuous measure have both "infinite $l^{2}$ masses": 1) always, $\sum_{k} u_{k}^{ \pm}(x)^{2}=\infty$ almost everywhere (in particular, there exist no positive frames, nor Riesz bases), but 2) $\sum_{k=1}^{n}\left(u_{k}^{+}(x)-u_{k}^{-}(x)\right)^{2}$ can grow "locally" as slow as we wish (for $n \longrightarrow \infty)$, and 3) it can happen that $\sum_{k=1}^{n} u_{k}^{-}(x)^{2}=o\left(\sum_{k=1}^{n} u_{k}^{+}(x)^{2}\right)$, and vice versa, as $n \longrightarrow \infty$ on a set of positive measure. Property 1 ) for the case of an orthonormal basis in $L^{2}(0,1)$ was settled earlier (V. Ya. Kozlov, 1948) using completely different (and more involved) arguments. Our elementary treatment includes also the case of unconditional bases in a variety of Banach spaces. For property 2), we show that, moreover, whatever is a monotone sequence $\epsilon_{k}>0$ satisfying $\sum_{k} \epsilon_{k}^{2}=\infty$ there exists an orthonormal basis $\left(u_{k}\right)_{k}$ in $L^{2}$ such that $\left|u_{k}(x)\right| \leq A(x) \epsilon_{k}, 0<A(x)<\infty$.
\end{abstract}

\section{The Subject. An introduction}

Let $(\Omega, \mu)$ be a measure space ( $\mu$ is not a finite sum of atoms), $L_{\mathbb{R}}^{2}(\Omega, \mu)$ be Lebesgue space of real valued functions and $\left(u_{k}\right)_{k \geq 1}$ a frame in $L_{\mathbb{R}}^{2}(\Omega, \mu)$. Recall that this means that the selfadjoint operator $S$ (the frame operator),

$$
S f=\sum_{k \geq 1}\left(f, u_{k}\right) u_{k}
$$

is an isomorphism on $L_{\mathbb{R}}^{2}(\Omega, \mu)$ : there exist $A>0, B>0$ such that $A \cdot I \leq S \leq B \cdot I$, that is

$$
A\|f\|^{2} \leq \sum_{k \geq 1}\left|\left(f, u_{k}\right)\right|^{2} \leq B\|f\|^{2} \quad \forall f \in L_{\mathbb{R}}^{2}(\Omega, \mu) .
$$

The right hand "half" of this condition is called the "Bessel sequence property"; its dual (equivalent) form is $\left\|\sum_{k \geq 1} c_{k} u_{k}\right\|^{2} \leq B \sum_{k \geq 1}\left|c_{k}\right|^{2}$ for every $c=\left(c_{k}\right)_{k \geq 1} \in l^{2}$ (look on the adjoint $T^{*}$ to $\left.T f=\left(\left(f, u_{k}\right)\right)_{k \geq 1}\right)$. Every Riesz basis (i.e., an isomorphic image of an orthonormal basis) is a bounded frame, and conversely, following the famous MarcusSpielman- Srivastava theorem [MSS2015], every bounded frame is a finite union of Riesz basis sequences (i.e., Riesz bases in their closed span).

Below, we consider the question on how can be distributed the signs $\operatorname{sign}\left(u_{k}(x)\right)$ of a frame for $k=1,2, \ldots$. For the case of orthonormal bases $\left(u_{k}\right)_{k \geq 1}$ the question was raised in [Koz1948]. Kozlov's result is as follows:

Let $\left(u_{k}\right)_{k \geq 1}$ be an orthonormal basis in $L_{\mathbb{R}}^{2}(0,1 ; d x)$ and $u_{k}^{ \pm}(x)=\max \left(0, \pm u_{k}(x)\right), x \in$ $(0,1)$ positive and negative parts of $u_{k}$, respectively. Then $\sum_{k} u_{k}^{+}(x)^{2}=\sum_{k} u_{k}^{-}(x)^{2}=\infty$ almost everywhere.

Kozlov's proof is quite involved and is based on topological properties of Lebesgue measure $d x$ on $(0,1)$. In [Koz1948], there are also some applications to uniqueness/divergence

2010 Mathematics Subject Classification. 42B20, 42B35, 47A30.

N. Nikolski is partially supported from RNF grant 14-41-00010 and the Chebyshev Lab, SPb University.

A. Volberg is partially supported by the NSF DMS-1600065. 
of Fourier series of $L^{2}$ functions with respect to general orthogonal bases. Later on, the same questions were discussed in [Aru1966], [Ovs1980]. We are also informed (thanks to D. Yakubovich, University Autonoma de Madrid) that the non-existence of positive Riesz bases was requested in the perceptive fields theory developed by V. D. Glezer and others, see for example [Gle2016]. After this paper appeared in arXiv (1812.06313 in math.FA), Prof. A.M.Powell kindly informed us on two more papers [JS2015] and [PS2016] where the question on positive bases in $L_{\mathbb{R}}^{p}(0,1)$ is also considered, see comments in 1.2(5) below.

1.1. Results. We give (simple) proofs to the following theorems.

Theorem 1.1. Let $\mu$ be a continuous measure (i.e., without point masses) and $\left(u_{k}\right)_{k \geq 1} a$ frame in $L_{\mathbb{R}}^{2}(\Omega, \mu)$. Then

$$
\sum_{k}\left(u_{k}^{+}(x)\right)^{2}=\sum_{k}\left(u_{k}^{-}(x)\right)^{2}=\infty, \quad \mu-\text { a.e. }
$$

In particular, there exists no positive frames (nor Riesz bases).

Theorem 1.1 is sharp in several senses: 1) first, one cannot weaken the frame condition of Theorem 1.1 up to "complete Bessel system" condition; 2) secondly, the signs of $u_{k}(x)$ are not "equidistributed" on subsequences of $\left(u_{k}\right)$ even for orthonormal bases; and 3) third, for sequence spaces $l$ strictly larger than $l^{2}$, the sequences $\left(u_{k}(x)\right)_{k \geq 1}$ can be in $l$ for every $x \in \Omega$. Precisely, the following facts hold.

Theorem 1.2. Let $(\Omega, \mu)$ be a measure space, $\mu$ a continuous measure.

I. There exists a sequence $\left(v_{n}\right)_{n \geq 1}$ in $L_{\mathbb{R}}^{2}(\Omega, \mu)$ such that (1) $v_{n} \geq 0$ on $\Omega$, (2) $\sum_{n} v_{n}(x)^{2}=$ $\infty$ on $\Omega$, (3) $0<\sum_{n}\left|\left(f, v_{n}\right)\right|^{2} \leq B\|f\|^{2}, \forall f \in L_{\mathbb{R}}^{2}(\Omega, \mu), f \neq 0$ (i.e., $\left(v_{n}\right)_{k \geq 1}$ is a complete Bessel sequence).

II. There exists a subset $E \subset \Omega, 0<\mu E<\infty$, and an orthonormal basis $\left(u_{k}\right)_{k \geq 1}$ in $L_{\mathbb{R}}^{2}(\Omega, \mu)$ such that $v_{n}:=u_{2 n} \mid E, n=1,2, \ldots$, satisfy conditions (1)-(3) of I ( $\Omega$ is replaced by $E)$.

Theorem 1.3. Let $\left\{b_{n}\right\}, b_{n}>0, \lim _{n} b_{n}=\infty$, be a monotone sequence such that

$$
\lim _{n} \frac{b_{n}}{b_{n-1}}=1
$$

and

$$
\sum \frac{1}{b_{n}}=\infty
$$

Then there exists a weight $w(x)>0$ on the real line $\mathbb{R}$ such that the orthonormal polynomials $p_{n}, n=0,1, \ldots$, form a basis in $L^{2}(\mathbb{R}, w d x)$ and

$$
\left|p_{n}(x)\right|^{2} \leq \frac{C(x)}{b_{n}}
$$

where $C(x)>0$ is locally bounded on $\mathbb{R}$. Notice that $\left|p_{n}(x)\right|^{2}=\left(p_{n}^{+}(x) \pm p_{n}^{-}(x)\right)^{2}=$ $p_{n}^{+}(x)^{2}+p_{n}^{-}(x)^{2}$.

The proof of Theorem 1.3 is given in the spirit of the spectral theory of Jacobi matrices, and heavily depends on methods developed by A. Máté and P. Nevai [MaN1983] and R. Szwarc [Szw2003], see more references and comments in Section 3 below. 
1.2. Comments. (1) For measures with point masses, no analog of Theorem 1.1 can be valid: there exist even orthogonal bases of nonnegative functions, for example, the natural basis in $l^{2}=L^{2}(\mathbb{N}$, count $)$.

(2) Also, in Theorem 1.1, the completeness property is essential, i.e. just for Riesz (or even orthonormal) sequences, nothing similar is true: the sequences $\left(u_{k}^{ \pm}(x)\right)_{k \geq 1}$ can even have finitely many non-zero coordinates only. Theorem 1.2 shows that keeping only "a half of frame conditions", namely that of complete Bessel systems, we loose the conclusion of 1.1: there exist positive complete Bessel sequences $\left(u_{k}\right)$ for which $\sum_{k} u_{k}(x)^{2}=\infty$ a.e.

(3) Theorem 1.2 implies also a kind of "non-equidistribution" of the signs in the family $\left(u_{k}\right)_{k}$ forming a frame (and even an orthonormal basis); see comments in Section 4.

(4) The sharpness of Theorem 1.1, as stated in Theorem 1.3, implies in particular, that taking $b_{n}=n$ we obtain an orthonormal polynomial basis $\left(u_{k}\right)_{k}$ in a weighted spaces $L^{2}(\mathbb{R}, w(x) d x), w(x)>0$, with the property $\left|u_{k}(x)\right| \leq \frac{c(x)}{k^{1 / 2}}$ for every $x \in \mathbb{R}$, and hence

$$
\sum_{k}\left|u_{k}(x)\right|^{2+\epsilon}<\infty \forall \epsilon>0 \quad \forall x \in \mathbb{R} .
$$

It is curious that it seems there exist no classical (or "semi-classical") orthonormal polynomials which show such kind asymptotic behavior. Indeed, in the classical setting, the best known estimates are shown by Laguerre orthonormal polynomial basis $L_{k}, k=0,1, \ldots$, in $L_{\mathbb{R}}^{2}\left(0, \infty ; e^{-x} d x\right)$, where we have

$$
L_{k}(x)=\frac{x^{-1 / 4} e^{x / 2}}{\sqrt{\pi} k^{1 / 4}} \operatorname{Cos}\left(2 \sqrt{k x}-\frac{\pi}{4}\right)+O\left(k^{-3 / 4}\right) \quad x>0,
$$

see [Sz1975], p.198), and hence $\sum_{k}\left|L_{k}(x)\right|^{4+\epsilon}<\infty(\forall \epsilon>0)$ almost everywhere, but $\left(L_{k}(x)\right)_{k \geq 0} \notin l^{4}$. Similar property holds for Hermite normalized polynomials in $L_{\mathbb{R}}^{2}\left(\mathbb{R} ; e^{-x^{2}} d x\right)$.

(5) The theme of the sign distribution of bases was developed at least in two other papers, [Aru1966] and [Ovs1980]. In [Aru1966], it is proved that for an unconditional basis $\left(u_{k}\right)_{k \geq 1}$ in $L_{\mathbb{R}}^{p}(0,1), \sum_{k} u_{k}^{ \pm}(x)^{p^{\prime}}=\infty$ a. e. if $2 \leq p<\infty, \frac{1}{p^{\prime}}+\frac{1}{p}=1$ (which contains Kozlov's theorem), and $\sum_{k} u_{k}^{ \pm}=\infty$ a.e. if $1<p<2$. (We will see in Section 2 that our elementary method entails these results and gives more). In [Ovs1980], a stronger property is proved under different hypotheses: if a sequence $\left(u_{k}\right) \subset L_{\mathbb{R}}^{2}(0,1)$ is normalized $\left\|u_{k}\right\|_{2}=1$, weakly tends to 0 and $\lim _{n} \int_{E}\left|u_{n}\right| d x>0$ for every $E \subset(0,1),|E|>0$, then $\sum_{k} u_{k}^{ \pm}(x)^{p}=\infty$ a.e. on $(0,1), \forall p<\infty$. Below, we show on a very simple example that, there exist positive uniformly minimal complete normalized sequences $\left(u_{k}\right) \subset L_{\mathbb{R}}^{2}(0,1)$, $u_{k} \geq 0$. In [PS2016], it is shown that there exists neither positive unconditional basis in $L_{\mathbb{R}}^{p}(0,1), 1 \leq p<\infty$ (already known from (Aru1966)), nor positive quasibasis; there are however positive Markushevich bases (minimal complete sequences having complete biorthogonal). In [JS2015] a positive Schauder basis in $L_{\mathbb{R}}^{1}(0,1)$ is constructed.

The rest of the paper is as follows: $\S 2$ - proof of theorem 1.1 and unconditional bases in Banach spaces, $\S 3$ - proof of theorem 1.3, and possible nonsymmetry between $u_{k}^{ \pm}, \S 4$ proof of theorem 1.2 .

Acknowledgements. The first author is highly grateful to Sasha and Olga Volberg, as well as to the Math Department of the MSU, organizing his short visit to Lansing-Ann Arbor (Fall 2018) with remarkable working conditions. He also recognizes a support from RNF grant 14-41-00010 and the Chebyshev Lab, SPb University. The second author is supported by NSF grant DMS 1600065. Both authors are grateful to Alexander Powell who indicated to them the papers [JS2015] and [PS2016]. 


\section{Proof of Theorem 1.1, And Signs of UnCONDItional BASES}

We start with a simplest version of our principal observation.

2.1. There exist no nonnegative Riesz bases in $L^{2}$. This result is not new, see [Aru1966], [PS2016]. However, seems that our proof is somewhat simpler.

Proof. Indeed, let $L^{2}=L_{\mathbb{R}}^{2}(\Omega, \mu), \mu$ continuous, $\mu \Omega<\infty$, and assume that $\left(u_{k}\right)$ is a normalized unconditional (= Riesz) basis having $u_{k} \geq 0$ on $\Omega$

and $f \in L_{\mathbb{R}}^{2}(\Omega, \mu)$. Using the development $f=\sum_{k>1}\left(f, u_{k}^{\prime}\right) u_{k}$ (where $\left(u_{k}^{\prime}\right)$ stands for the dual sequence, $\left.\left(u_{k}, u_{j}^{\prime}\right)=\delta_{k j}\right)$, define $R_{N} f=\sum_{k \geq N}\left(f, u_{k}^{\prime}\right) u_{k}$ and observe that

$$
\begin{gathered}
\left\|R_{N} f\right\|_{L^{1}} \leq \int_{\Omega} \sum_{k \geq N}\left|\left(f, u_{k}^{\prime}\right)\right| u_{k}=\sum_{k \geq N}\left|\left(f, u_{k}^{\prime}\right)\right|\left(u_{k}, 1\right)_{L^{2}}= \\
\left(f_{*}, R_{N}^{*} 1\right)_{L^{2}}, \text { where } \mathrm{f}_{*}=\sum_{\mathrm{k} \geq 1}\left|\left(\mathrm{f}, \mathrm{u}_{\mathrm{k}}^{\prime}\right)\right| \mathrm{u}_{\mathrm{k}} .
\end{gathered}
$$

Since $\left\|f_{*}\right\|_{2} \leq B\|f\|_{2}$, it means $\left\|R_{N}: L^{2} \longrightarrow L^{1}\right\|_{L^{1}} \leq B\left\|R_{N}^{*} 1\right\|_{2}$. But $\lim _{N}\left\|R_{N}^{*} 1\right\|_{2}=0$, and the $\operatorname{map} S_{N} f=f-R_{N} f$ has a finite rank, so we get that $i d: L^{2} \longrightarrow L^{1}$ is compact, which is not the case (for example, if $\mu \Omega=1$, there exists a unimodular orthonormal sequences in $L^{2}$ ).

\subsection{Remarks on other spaces.}

Let $L^{p}=L_{\mathbb{R}}^{p}(\Omega, \mu), \mu$ continuous, $\mu \Omega<\infty$.

(1) Exactly the same lines (with $\left\|f_{*}\right\|_{2}$ replaced by $\left\|f_{*}\right\|_{X}$, and $\left\|R_{N}^{*} 1\right\|_{2}$ by $\left\|R_{N}^{*} 1\right\|_{X^{*}}$ ) show that there is no nonnegative unconditional bases in any reflexive Banach space $X$ of measurable functions such that $L^{\infty}(\mu) \subset X^{*} \subset L^{1}(\mu), X^{*}$ stands for the dual space with respect to the duality $(f, h)=\int_{\Omega} f \bar{h} d \mu$. Example: $X=L_{\mathbb{R}}^{p}(\Omega, \mu), 1<p<\infty$. Later on, we return to $L^{p}$ spaces in order to consider the sign distributions of unconditional bases in more details (see point 2.5 below).

(2) One can slightly strengthen property 2.1 replacing the condition $u_{k}(x) \geq 0$ a.e. for $\max _{j} h_{j}(x) u_{k}(x) \geq 0$ a.e. $(\forall k)$ where $\left\{h_{j}\right\}$ stands for a finite family of functions taking values \pm 1 .

Now, we turn to theorem 1.1 whose proof depends on the following elementary lemma and some easy properties of compact operators.

\subsection{The tale of two lemmas.}

Lemma 2.1. Let $L_{\mathbb{R}}^{2}(\Omega, \mu)$ as before, $E \subset \Omega$ with $0<\mu E<\infty$, and $\left(v_{k}\right)_{k \geq 1}$ a sequence in $L_{\mathbb{R}}^{2}(\Omega, \mu)$ such that

$$
\sum_{k \geq 1}\left|v_{k}(x)\right|^{2} \leq M^{2} \text { for } x \in E .
$$

Then, (1) the map $V: f \longmapsto\left(\left(f, v_{k}\right)\right)_{k \geq 1}$ is compact as $L^{2}(E, \mu) \longrightarrow l^{2}$, and (2) the map $V^{*}:\left(c_{k}\right)_{k \geq 1} \longmapsto \sum_{k \geq 1} c_{k} v_{k} \mid E$ is compact $l^{2} \longrightarrow L^{2}(E, \mu)$ as well. 
Proof. (1) Writing $V=V_{N}+V_{N}^{\prime}$, where

$$
V_{N} f=\left(\left(f, v_{1}\right), \ldots,\left(f, v_{N}\right), 0,0, \ldots\right),
$$

we get for every $c=\left(c_{k}\right)_{k \geq 1} \in l^{2}, f \in L^{2}(E)$ and $N \geq 1$,

$$
\begin{gathered}
\left|\left(V_{N}^{\prime} f, c\right)\right|=\left|\sum_{k \geq N} c_{k}\left(f, v_{k}\right)\right| \leq\|f\|_{2} \sum_{k \geq N}\left|c_{k}\right| \cdot\left\|v_{k} \mid E\right\|_{2} \leq \\
\|f\|_{2}\|c\|_{2}\left(\sum_{k \geq N}\left\|v_{k} \mid E\right\|_{2}^{2}\right)^{1 / 2}=:\|f\|_{2}\|c\|_{2} \cdot \epsilon_{N} .
\end{gathered}
$$

Hence $\left\|V_{N}^{\prime}\right\| \leq \epsilon_{N}$, where $\epsilon_{N} \longrightarrow 0$ since $\epsilon_{1} \leq M(\mu E)^{1 / 2}<\infty$. The claim follows.

$V^{*}$ is the adjoint of $V$ of point (1).

Lemma 2.2. Let $\left(v_{k}\right)_{k \geq 1}$ and $E \subset \Omega$ be as in Lemma 2.1 and $\left(u_{k}\right)_{k \geq 1}$ a frame in $L_{\mathbb{R}}^{2}(\Omega, \mu)$. Then, the operators

$$
U f=\sum_{k \geq 1}\left(f, v_{k}\right) u_{k} \text { acting as } L^{2}(E, \mu) \longrightarrow L_{\mathbb{R}}^{2}(\Omega, \mu)
$$

its adjoint $U^{*} f=\sum_{k \geq 1}\left(f, u_{k}\right) v_{k}$, and $U^{\prime}$, given by

$$
U^{\prime} f=\sum_{k \geq 1}\left(f, v_{k}\right) v_{k} \mid E: L^{2}(E, \mu) \longrightarrow L_{\mathbb{R}}^{2}(E, \mu)
$$

are compact.

Proof. For $U$, the frame definition entails $\|U f\|_{2}^{2} \leq B\|V f\|_{l^{2}}^{2}$ for every $f \in L^{2}(E, \mu)$, and the claim follows from Lemma 2.1. For the operator $U^{\prime}$, we repeat the estimate of Lemma 2.1:

$$
\left|\sum_{k \geq N}\left(f, v_{k}\right) v_{k}\right|^{2} \leq\left(\sum_{k \geq N}\left|\left(f, v_{k}\right)\right|^{2}\right)\left(\sum_{k \geq N}\left|v_{k}\right|^{2}\right),
$$

which gives the result after integration over $E$.

\subsection{Proof of the Theorem 1.1.}

Proof. Suppose $\sum_{k \geq 1}\left(u_{k}^{-}(x)\right)^{2}<\infty$ on a set of positive measure. Then there exist $E \subset \Omega$ and $M>0$ such that

$$
\sum_{k \geq 1}\left(u_{k}^{-}(x)\right)^{2} \leq M^{2} \quad \forall x \in E \text { and } 0<\mu E<\infty .
$$

This implies the same contradiction as in point 2.1 that the natural embedding $L^{2}(E, \mu) \hookrightarrow$ $L^{1}(E, \mu)$ is compact. The steps are as follows.

(1) Setting $v_{k}=u_{k}^{-}$, we have from Lemma 2.1

$$
\|V f\|_{l^{2}}^{2}=\sum_{k \geq 1}\left|\left(f, u_{k}^{-}\right)\right|^{2} \leq\|V\|^{2} \cdot\|f\|^{2} \text { on } L^{2}(E, \mu)
$$

and from the frame definition $\sum_{k \geq 1}\left|\left(f, u_{k}\right)\right|^{2}=(S f, f) \leq B\|f\|_{2}^{2}\left(\forall f \in L^{2}(\Omega, \mu)\right)$. Hence

$$
\sum_{k \geq 1}\left|\left(f, u_{k}^{+}\right)\right|^{2} \leq C^{2}\|f\|^{2} \text { on } L^{2}(E, \mu), C^{2} \leq 2\left(\|V\|^{2}+B\right) \text {. }
$$

(2) It follows from $u_{k}^{+}=u_{k}+u_{k}^{-},(1)$ and Lemma 2.2 that $W$,

$$
W f:=\sum_{k \geq 1}\left(f, u_{k}^{+}\right) u_{k}^{-} \mid E
$$

acting as $L^{2}(E, \mu) \longrightarrow L_{\mathbb{R}}^{2}(E, \mu)$ is compact. 
(3) Now, the quadratic form $(S f, f)=(U f, f)+(W f, f)+(X f, f)$ on $L^{2}(E, \mu)$, where

$$
X f:=\sum_{k \geq 1}\left(f, u_{k}^{+}\right) u_{k}^{+}
$$

is equivalent to $(f, f)=\|f\|^{2}$ (in the sens $A\|f\|^{2} \leq(S f, f) \leq B\|f\|^{2}$ ), and the forms $(U f, f)$ and $(W f, f)$ are compact on $L^{2}(E, \mu)$. It implies that $(X f, f)$ is equivalent to $\|f\|^{2}$ on a subspace $H \subset L^{2}(E, \mu)$ of finite co-dimention.

(4) The latter property means that the compression

$$
X_{E}: L^{2}(E, \mu) \longrightarrow L^{2}(E, \mu), \quad X_{E} f=X f \mid E, f \in L^{2}(E, \mu
$$

is Fredholm. Let $R: L^{2}(E, \mu) \longrightarrow L^{2}(E, \mu)$ be a regularizer of $X_{E}$, a bounded operator such that

$$
R X_{E}=i d+K \text { where } K: L^{2}(E, \mu) \longrightarrow L^{2}(E, \mu) \text { is compact. }
$$

(5) Show that $X_{E}: L^{2}(E, \mu) \longrightarrow L^{1}(E, \mu)$ is compact. Indeed, similarly to Lemmas 2.1 and 2.2 , the norms $\left\|X_{E, N}: L^{2}(E, \mu) \longrightarrow L^{1}(E, \mu)\right\|$ tends to zero as $N \longrightarrow \infty$, where $X_{E, N} f=\sum_{k \geq N}\left(f, u_{k}^{+}\right) u_{k}^{+} \mid E$. In fact,

$$
\begin{aligned}
& \left\|\sum_{k \geq N}\left(f, u_{k}^{+}\right) u_{k}^{+}\right\|_{L^{1}(E, \mu)} \leq \sum_{k \geq N}\left|\left(f, u_{k}^{+}\right)\right| \cdot\left\|u_{k}^{+}\right\|_{L^{1}(E, \mu)}= \\
& \sum_{k \geq N}\left|\left(f, u_{k}^{+}\right)\right| \cdot\left(u_{k}^{+}, 1\right)_{L^{2}(E, \mu)} \leq \\
& \leq\left(\sum_{k \geq N}\left|\left(f, u_{k}^{+}\right)\right|^{2}\right)^{1 / 2}\left(\sum_{k \geq N}\left|\left(u_{k}^{+}, 1\right)_{L^{2}(E, \mu)}\right|^{2}\right)^{1 / 2} \leq \\
& C\|f\|\left(\sum_{k \geq N}\left|\left(u_{k}^{+}, 1\right)_{L^{2}(E, \mu)}\right|^{2}\right)^{1 / 2}:=C\|f\| \epsilon_{N},
\end{aligned}
$$

and $\lim _{N} \epsilon_{N}=0$ in view of (1) above. Now, regarding the identity $R X_{E}=i d+K$ as acting from $L^{2}(E, \mu)$ to $L^{1}(E, \mu)$, we get that the natural embedding $i d: L^{2}(E, \mu) \hookrightarrow$ $L^{1}(E, \mu)$ is compact which contradicts to $\mu E>0$.

2.5. Sign distributions for bases in more general spaces. Here we give "an abstract version" of the reasoning from 2.1-2.4 (without trying to find the most general setting). Let as before, $(\Omega, \mu)$ be a measure space with a continuous measure, and (WLOG) $\mu \Omega<\infty$. $X$ will be a real reflexive Banach lattice of measurable functions such that

$$
L^{\infty} \subset X \subset L^{1} \text { and } X^{*}=\left\{h: h f \in L^{1}, \forall f \in X\right\}
$$

with the duality $(f, h)=\int_{\Omega} f h d \mu$.

Example: $X=L_{\mathbb{R}}^{p}(\Omega, \mu), 1<p<\infty$, or $X$ is a (rearrangement invariant) symmetric space of measurable functions, see [KPS1978]. Let $U=\left(u_{k}\right)_{k \geq 1}$ be a normalized unconditional basis in $X, U^{\prime}=\left(u_{k}^{\prime}\right)_{k \geq 1}$ the dual basis, $\left(u_{k}, u_{j}^{\prime}\right)=\delta_{k j}$, so that

$$
f=\sum_{k \geq 1}\left(f, u_{k}^{\prime}\right) u_{k} \text { for every } f \in X .
$$

Denote

$$
\text { Coef }(U)=\left\{c(f):=\left\{\left(f, u_{k}^{\prime}\right)\right\}: f \in X\right\}
$$

the sequence space of coefficients (if needed we will add the space to the notation: $\operatorname{Coe} f(U, X)$ ); this is a sequence lattice, $\left(a_{k}\right) \in \operatorname{Coef}(U) \Rightarrow\left(\lambda_{k} a_{k}\right) \in \operatorname{Coef}(U), \forall\left(\lambda_{k}\right) \in l^{\infty}$, where the standard $0-1$ sequences form an unconditional basis. Clearly, $\operatorname{Coef}\left(U^{\prime}\right)=(\operatorname{Coe} f(U))^{*}$ (with respect to the duality $\left.(a, b)=\sum_{k>1} a_{k} b_{k}\right)$. With this notation, here is our claim on the sign distributions. 
Theorem 2.3. Let $X$ be a reflexive Banach lattice of measurable functions satisfying the above conditions, and $U=\left(u_{k}\right)$ be a normalized unconditional basis in $X$. Then, for every $E \subset \Omega, \mu E>0$,

$$
\left(\int_{E} u_{k}^{+} d \mu\right)_{k \geq 1} \notin(\operatorname{Coef}(U))^{*} \text { and }\left(\int_{\mathrm{E}} \mathrm{u}_{\mathrm{k}}^{-} \mathrm{d} \mu\right)_{\mathrm{k} \geq 1} \notin(\operatorname{Coef}(\mathrm{U}))^{*} .
$$

Proof. Here is the proof of Theorem 2.3. The reasoning repeats our steps above. Namely, let

$$
V^{ \pm} f=\sum_{k \geq 1}\left(f, u_{k}^{\prime}\right) u_{k}^{ \pm}, \text {so that } i d=V^{+}-V^{-}
$$

and

$$
V_{N}^{ \pm} f=\sum_{k \geq N}\left(f, u_{k}^{\prime}\right) u_{k}^{ \pm}, f \in X, N=1,2, \ldots
$$

Now, assuming $R^{-} u:=\left(\int_{E} u_{k}^{-} d \mu\right)_{k \geq 1} \in(\operatorname{Coef}(U))^{*}$ for some $E, \mu E>0$, we obtain

$$
\left\|V_{N}^{-} f\right\|_{1} \leq \sum_{k \geq N}\left|\left(f, u_{k}^{\prime}\right)\right| \int_{E} u_{k}^{-} d \mu=\left(c\left(f_{*}\right), R_{N}^{-} u\right),
$$

where $f_{*}=\sum_{k \geq 1}\left|\left(f, u_{k}^{\prime}\right)\right| u_{k}$ (with $\left\|f_{*}\right\|_{X} \leq C\|f\|_{X}$, unconditional basis) and $R_{N}^{-} u=$ $\left\{0, \ldots 0, \int_{E} u_{N+1}^{-} d \mu, \ldots\right\}$. Since $\left(u_{k}^{\prime}\right)$ is a basis in $X^{*}$ (reflexivity of $\left.X\right)$, we get

$$
\left\|V_{N}^{-}: X \longrightarrow L^{1}\right\| \leq C\left\|R_{N}^{-} u\right\|_{X^{*}} \longrightarrow 0 \text { as } N \longrightarrow \infty .
$$

The same for $V_{N}^{+}$:

$$
\left\|V_{N}^{+} f\right\|_{1} \leq \sum_{k \geq N}\left|\left(f, u_{k}^{\prime}\right)\right| \int_{E} u_{k}^{+} d \mu=\left(c\left(f_{*}\right), R_{N} u-R_{N}^{-} u\right),
$$

where $R_{N} u=\left\{0, \ldots 0, \int_{E} u_{N+1} d \mu, \ldots\right\}$, and hence

$$
\left\|V_{N}^{+}: X \longrightarrow L^{1}\right\| \leq C\left\|R_{N} u-R_{N}^{-} u\right\|_{X^{*}} \leq C\left(\left\|R_{N} u\right\|_{X^{*}}+\left\|R_{N}^{-} u\right\|_{X^{*}}\right) .
$$

But $\int_{E} u_{k} d \mu=\left(\chi_{E}, u_{k}\right)$ and hence $\left(\int_{E} u_{k} d \mu\right)_{k \geq 1} \in(\operatorname{Coef}(U))^{*}\left(X \subset L^{1}\right.$, and so $L^{\infty} \subset$ $\left.X^{*}\right)$. It implies $\lim _{N}\left\|R_{N} u\right\|_{X^{*}}=0$, and as above, we conclude that both $V^{+}, V^{-}: X \longrightarrow$ $L^{1}$ are compact operators and $i d=V^{+}-V^{-}$. But there exists a unimodular sequence $v_{n}$ in $X$ which tends weakly to zero (it is clear when replacing $(\Omega, \mu)$ by isomorphic measure space $((0,1, d x))$, but $\left\|v_{n}\right\|_{1}=\mu \Omega>0$. Contradiction.

2.6. Now we give an application of Theorem 2.3. (1) Type, cotype, and unconditional bases. Recall (see for example [Woj1996], point III.A.17) that a Banach space $X$ is said to have cotype $q, 2 \leq q \leq \infty$, if for some constant $C>0$ and for every finite sequence $x=\left(x_{j}\right), x_{j} \in X$,

$$
C \int_{0}^{1}\left\|\sum_{j} r_{j}(t) x_{j}\right\| d t \geq\|x\|_{l^{q}}:=\left(\sum_{j}\left\|x_{j}\right\|^{q}\right)^{1 / q},
$$

and it has type $q, 1 \leq q \leq 2$, if

$$
\int_{0}^{1}\left\|\sum_{j} r_{j}(t) x_{j}\right\| d t \leq C\|x\|_{l^{q}}
$$


where $\left(r_{j}\right)_{j \geq 1}$ stands for the sequence of Rademacher functions. It is known (and is proved in [Woj1996], Ch. III.A) that $X$ has type $q$ if and only if $X^{*}$ has cotype $q^{\prime}, \frac{1}{q^{\prime}}+\frac{1}{q}=1$, and if $X$ has type $q_{1} \leq 2$ and a cotype $q_{2} \geq 2$ and if $U=\left(u_{k}\right)$ is a normalized unconditional basis in $X$ then

$$
l^{q_{1}} \subset \operatorname{Coef}(U, X) \subset l^{q_{2}} .
$$

Corollary. If in condition of Theorem 2.3, the lattice $X$ has a cotype $q_{2}$ then

$$
\left(\int_{E} u_{k}^{ \pm} d \mu\right)_{k \geq 1} \notin l^{q_{2}^{\prime}}(\forall E \subset \Omega, \mu E>0) \text {, whence } \sum_{k \geq 1}\left(u_{k}^{ \pm}(x)\right)^{q_{2}^{\prime}}=\infty \text { a.e. } \Omega \text {. }
$$

Indeed, $l^{q_{2}^{\prime}} \subset \operatorname{Coe} f(U, X)^{*}$, and the first claim follows from the theorem. Also

$$
\left(\int_{E} u_{k}^{ \pm} d \mu\right)^{q_{2}^{\prime}} \leq c \int_{E}\left(u_{k}^{ \pm}\right)^{q_{2}^{\prime}} d \mu
$$

whence $\int_{E} \sum_{k}\left(u_{k}^{ \pm}\right)^{q_{2}^{\prime}} d \mu=\infty$ for every $E, \mu E>0$, which is equivalent to

$$
\sum_{k \geq 1}\left(u_{k}^{ \pm}(x)\right)^{q_{2}^{\prime}}=\infty \quad \text { a.e. } \Omega
$$

(2) The spaces $X=L_{\mathbb{R}}^{p}(\Omega, \mu)$. It is known (and is basically equivalent to Khintchin's inequality, see [Woj1996], point III.A.22) that $L^{p}$ is of type $q_{1}=\min (2, p)$ and of cotype $q_{2}=\max (2, p)$, and hence

$$
\operatorname{Coef}\left(U, L^{p}\right) \subset l^{q} \text {, where } q=\max (2, p) .
$$

(It is curious to note how different is the coefficient space for the standard trigonometric Schauder basis of $L^{p}(0,2 \pi)$ : the Hausdorff-Young inequality tells that $\operatorname{Coe} f\left(e^{i n x}, L^{p}\right) \subset$ $l^{p^{\prime}}$ for $1<p \leq 2$ and $\operatorname{Coef}\left(e^{i n x}, L^{p}\right) \subset l^{2}$ for $\left.p \geq 2\right)$.

Corollary. Let $X=L_{\mathbb{R}}^{p}(\Omega, \mu), 1<p<\infty$, and $U=\left(u_{k}\right)$ a normalized unconditional basis in $X$. Then for every $E \subset \Omega, \mu E>0$, we have

$$
\begin{aligned}
& \text { for } 1<p \leq 2,\left(\int_{E} u_{k}^{ \pm} d \mu\right)_{k \geq 1} \notin l^{2}, \text { and in particular } \sum_{k \geq 1} u_{k}^{ \pm}(x)^{2}=\infty \text { a.e., } \\
& \text { for } 2<p<\infty,\left(\int_{E} u_{k}^{ \pm} d \mu\right)_{k \geq 1} \notin l^{p^{\prime}}, \text { and in particular } \sum_{k \geq 1} u_{k}^{ \pm}(x)^{p^{\prime}}=\infty \text { a.e., }
\end{aligned}
$$

where $\frac{1}{p^{\prime}}+\frac{1}{p}=1$.

The necessary condition $\left(u_{k}^{ \pm}(x)\right)_{k \geq 1} \notin l^{p^{\prime}}$ a.e. for $p \geq 2$, as well as a weaker condition $\left(u_{k}^{ \pm}(x)\right)_{k \geq 1} \notin l^{1}$ a.e. for $1<p<2$, were found already in [Aru1966].

\section{Pointwise Behavior of orthogonal polynomials, And proof of Theorem}

$$
1.3 \text {. }
$$

Here we show that the exponent 2 in Theorem 1.1 cannot be improved: for every $\epsilon_{k} \searrow 0$ having $\sum_{k} \epsilon_{k}^{2}=\infty$, there exists an orthonormal basis $\left(u_{k}\right)$ with $\left|u_{k}(x)\right| \leq C(x) \epsilon_{k}$ a.e.; in particular, taking $\epsilon_{k}=(k+1)^{-1}$, we get $\sum_{k>1}\left|u_{k}(x)\right|^{2+\epsilon}<\infty$ a.e $(\forall \epsilon>0)$. Theorem 1.3 is a simple restating of Theorem 3.1 below. The proof of Theorem 3.1 is based on the three terms recurrence for orthogonal polynomials but its direct application (replacing 
moduli of sums by sums of moduli) fails. Instead, we use a subtle reasoning introduced in a similar situation in important papers by A. Máté and P. Nevai [MaN1983] and R.Szwarc [Szw2003]. The basic facts of the theory of orthogonal polynomials are contained (for example) in the books [Sz1975], [Ber1968], [Sim2005]. One of them, the classical J. Favard theorem (1935), claims that whatever are real sequences $b_{k} \in \mathbb{R}$ and $a_{k}>0$ and the sequence of polynomials $p_{k}, \operatorname{deg}\left(p_{k}\right)=k, k=0,1, \ldots$ defined by the three term recurrence

$$
x p_{k}(x)=a_{k+1} p_{k+1}(x)+b_{k} p_{k}(x)+a_{k} p_{k-1}(x), \quad k=0,1,2, \ldots,
$$

where $p_{0}=1, p_{-1}(x)=0$, there exists (at least one) Borel measure $\mu \geq 0$ on the real line such that $p_{k} \in L^{2}(\mu)(\forall k \geq 0)$ and $\left(p_{k}, p_{j}\right)_{L^{2}(\mu)}=\delta_{k, j}$ (Kronecker delta).

In fact, the measure $\mu$ is the scalar spectral measure of the associated tridiagonal (selfadjoint) Jacobi matrix $J$ having $\left(b_{k}\right)_{k \geq 0}$ on the main diagonal and $\left(a_{k}\right)_{k \geq 1}$ on two side diagonals.

Another classical theorem (T. Carleman) tell us that such a measure is unique if $\sum_{k \geq 0} \frac{1}{a_{k}}=\infty$ (the so-called "determined case") - the condition is obviously satisfied in case of Theorem 3.1 below. It follows that the polynomials are dense in $L^{2}(\mu)$, and hence $\left(p_{k}\right)_{k \geq 0}$ forms an orthonormal basis in $L^{2}(\mu)$. A huge theory of orthogonal polynomials and the associated Jacobi matrices is (partially) presented in books mentioned above.

We use here the work of R. Szwarc [Szw2003]. We just repeat several calculations from this article to get the following result.

Theorem 3.1. Let $\left\{b_{n}\right\}, b_{n}>0, b_{n} \rightarrow \infty$, be a monotone sequences such that $b_{n} / b_{n-1} \rightarrow$ 1 , and $\sum b_{n}^{-1}=\infty$ and let $a_{n}$ be such that $a_{n}=\frac{1}{2 B} \sqrt{b_{n} b_{n-1}}$, where $0<B<1$. Then the Jacobi matrix with $\left\{b_{n}\right\}$ on the main diagonal and $\left\{a_{n}\right\}$ on two other diagonals will have absolutely continuous spectrum and the orthogonal polynomials $\left\{p_{n}\right\}$ will have a local uniform estimate

$$
\left|p_{n}(x)\right|^{2} \leq C b_{n}^{-1}
$$

Here is the theorem from [Szw2003].

Theorem 3.2. Assume the sequences $a_{n}$ and $b_{n}$ satisfy $a_{n} \rightarrow \infty, b_{n} \rightarrow \infty, b_{n} / b_{n-1} \rightarrow 1$ and $a_{n}^{2} / b_{n} b_{n-1} \rightarrow 1 / 4 B^{2}>1 / 4$. Let the sequences

$$
\frac{a_{n}^{2}}{b_{n} b_{n-1}}, \frac{\left(b_{n}+b_{n-1}\right)}{a_{n}^{2}}, \frac{1}{a_{n}^{2}}
$$

have bounded variation. Then the corresponding Jacobi matrix $J$ with $b_{n}$ on the main diagonal is essentially self-adjoint if and only if $\sum a_{n}^{-1}=\infty$. In that case the spectrum of $J$ coincides with the whole real line and the spectral measure is absolutely continuous.

Theorem 3.1 follows from this claim (except for the estimates of polynomials) immediately as the monotonicity of $\left\{b_{n}\right\}$ ensures all the regularity required in Theorem 3.2 , and, of course, in assumptions of Theorem $3.1 \sum b_{n}^{-1}=\infty$ gives $\sum a_{n}^{-1}=\infty$.

Let us follow Szw2003 to show the estimate on orthogonal polynomials with respect to the spectral measure of $J$. There are several non essential typos in [Szw2003], and we will correct them on the way.

We have

$$
x p_{n}(x)=a_{n+1} p_{n+1}(x)+b_{n} p_{n}(x)+a_{n} p_{n-1}(x) .
$$

We put

$$
A_{n}(x):=p_{n}(x) \sqrt{b_{n}-x}, \quad n \geq N, \Lambda_{n}:=B \frac{a_{n}}{\sqrt{\left(b_{n}-x\right)\left(b_{n-1}-x\right)}}
$$


With this notation (3.1) becomes

$$
0=\Lambda_{n+1} A_{n+1}(x)+B A_{n}(x)+\Lambda_{n} A_{n-1}(x) .
$$

By assumptions, $\Lambda_{n} \rightarrow \frac{1}{2}$ and $B<1$. Moreover, since

$$
B^{2} \Lambda_{n}^{-2}=\frac{b_{n} b_{n-1}}{a_{n}^{2}}-\frac{\left(b_{n}+b_{n-1}\right)}{a_{n}^{2}} x+\frac{1}{a_{n}^{2}} x^{2},
$$

it is of bounded variation, and thus so is $\Lambda_{n}$.

Theorem 3.3. (Maté, Nevai, MaN1983] ) Let $\Lambda_{n}(x)$ be a positive valued sequence whose terms depend continuously on $x \in[a, b]$. Let $A_{n}(x)$ be a real valued sequence of continuous functions satisfying (3.3) for $n \geq N$. Assume the sequence $\Lambda_{n}(x)$ has bounded variation and $\Lambda_{n}(x) \rightarrow \frac{1}{2}$ for $x \in[a, b]$. Let $|B|<1$. Then there is a strictly positive function $f(x)$ continuous on $[a, b]$ such that

$$
A_{n}^{2}(x)-A_{n-1}(x) A_{n+1}(x) \rightarrow f(x)
$$

uniformly for $x \in[a, b]$. Moreover, there is a constant $c$ such that

$$
\left|A_{n}(x)\right| \leq c
$$

for $n \geq 0$ and $x \in[a, b]$.

Clearly to prove Theorem 3.1 it is sufficient to use this result of Maté, Nevai. Indeed, (3.5) obviously gives us the bound on $p_{n}(x)^{2}$ stated in Theorem 3.1. For the readers convenience and for making the paper self-contained we give a proof to Theorem 3.3 .

Proof. To prove Theorem 3.3, one first uses recurrent relation (3.3) to write

$$
A_{n-1}=-\frac{\Lambda_{n+1}}{\Lambda_{n}} A_{n+1}(x)-\frac{B}{\Lambda_{n}} A_{n}(x)
$$

and, hence,

$$
A_{n}^{2}-A_{n-1} A_{n+1}=A_{n}^{2}+\frac{\Lambda_{n+1}}{\Lambda_{n}} A_{n+1}^{2}+\frac{B}{\Lambda_{n}} A_{n} A_{n+1}
$$

This can be rewritten as follows

$$
A_{n}^{2}-A_{n-1} A_{n+1}=\left(A_{n}+\frac{B}{2 \Lambda_{n}} A_{n+1}\right)^{2}+\left(\frac{\Lambda_{n+1}}{\Lambda_{n}}-\frac{B^{2}}{4 \Lambda_{n-1}^{2}}\right) A_{n+1}^{2}
$$

Now we combine that equality with the facts that $\Lambda_{n} \rightarrow \frac{1}{2}$ and $B<1$, and this combination implies the following estimate:

$$
A_{n+1}^{2} \leq C\left(A_{n}^{2}-A_{n-1} A_{n+1}\right) .
$$

But we can also rewrite the equality (3.6) in another form:

$$
A_{n}^{2}-A_{n-1} A_{n+1}=\frac{\Lambda_{n+1}}{\Lambda_{n}}\left(A_{n+1}+\frac{B}{2 \Lambda_{n+1}} A_{n}\right)^{2}+\left(1-\frac{B^{2}}{4 \Lambda_{n} \Lambda_{n+1}}\right) A_{n}^{2}
$$

This formula and the same two facts that $\Lambda_{n} \rightarrow \frac{1}{2}$ and $B<1$ imply now the following estimate:

$$
A_{n}^{2} \leq C\left(A_{n}^{2}-A_{n-1} A_{n+1}\right)
$$

Let us also write

$$
A_{n+2}=-\frac{B}{\Lambda_{n+2}} A_{n+1}-\frac{\Lambda_{n+1}}{\Lambda_{n+2}} A_{n}
$$


That equality together with (3.6) give us the following:

$$
\begin{aligned}
& \left(A_{n+1}^{2}-A_{n} A_{n+2}\right)-\left(A_{n}^{2}-A_{n-1} A_{n+1}\right)=\left(1-\frac{\Lambda_{n+1}}{\Lambda_{n}}\right) A_{n+1}^{2}+ \\
& B\left(\frac{1}{\Lambda_{n+2}}-\frac{1}{\Lambda_{n}}\right) A_{n} A_{n+1}+\left(\frac{\Lambda_{n+1}}{\Lambda_{n+2}}-1\right) A_{n}^{2} .
\end{aligned}
$$

Denoting $\Delta_{n}:=A_{n}^{2}-A_{n-1} A_{n+1}$ we get from (3.8), (3.10) and (3.11):

$$
\Delta_{n}>0, A_{n}^{2}+A_{n+1}^{2} \leq C \Delta_{n}, \quad\left|\Delta_{n+1}-\Delta_{n}\right| \leq C\left(\left|\Lambda_{n+1}-\Lambda_{n}\right|+\left|\Lambda_{n+1}-\Lambda_{n+2}\right|\right) \Delta_{n} .
$$

Denote $\varepsilon_{n}:=\left|\Lambda_{n+1}-\Lambda_{n}\right|+\left|\Lambda_{n+1}-\Lambda_{n+2}\right|$. Then

$$
\left(1-C \varepsilon_{n}\right) \Delta_{n} \leq \Delta_{n+1} \leq\left(1+C \varepsilon_{n}\right) \Delta_{n},
$$

and $\sum \varepsilon_{n}$ converges by the assumption that $\Lambda_{n}$ has bounded variation.

Therefore, $\Delta_{n}$ uniformly converges to a strictly positive function $f$, and hence, $A_{n}^{2}$ are uniformly bounded uniformly bounded for $n>N(x)$ (namely, by a multiple $C f(x)$ of $f(x)$ ). Thus (3.5) is proved, Theorem 3.3 of Maté-Nevai is proved, and we already said that this proves the bound of Theorem 3.1 .

\section{Counterexamples: an attempt on Bessel systems, and proof of THEOREM 1.2.}

4.1. Part I of the Theorem 1.2. A natural question whether a "half of the frame condition", namely the Bessel one, is sufficient for getting the conclusion of theorem 1.1, is essentially equivalent (in the notation of Theorem 1.2) to the following: whether

$$
(1) \&(2) \Rightarrow\left(3^{\prime}\right):=\exists f \in L_{\mathbb{R}}^{2}(\Omega, \mu) \text { such that } \sum_{\mathrm{n}}\left|\left(\mathrm{f}, \mathrm{v}_{\mathrm{n}}\right)\right|^{2}=\infty ?
$$

Indeed, if (3') does not hold (and we have $\sum_{n}\left|\left(f, v_{n}\right)\right|^{2}<\infty, \forall f \in L_{\mathbb{R}}^{2}(\Omega, \mu)$ ), we automatically get property (3) of theorem 1.3 just due to Banach-Steinhaus theorem applied to the semi-norms

$$
p_{n}(f)=\left(\sum_{k=1}^{n}\left(f, v_{k}\right)^{2}\right)^{1 / 2} .
$$

However, there is a counterexample which gives a negative answer to this question and proves Part I of the Theorem 1.2.

4.2. Counterexample. Let $(\Omega, \mu)=(0,1), d x$, and $\left(v_{k}\right)_{k \geq 1}$ be any enumeration of the indicator functions $\chi_{I}$ of dyadic subintervals $\mathcal{D}=\left\{I=I_{j, n}\right\}$ of $(0,1)$ :

$$
I_{j, n}=\left(\frac{j}{2^{n}}, \frac{j+1}{2^{n}}\right), j=0, \ldots, 2^{n}-1 .
$$

Properties (1) and (2), as well as the completeness of $\left(v_{k}\right)$, are obvious. For (3), we write

$$
\sum_{k}\left|\left(f, v_{k}\right)\right|^{2}=\sum_{I \in \mathcal{D}}\left(\frac{1}{|I|} \int_{I} f d x\right)^{2}|I|^{2},
$$

and notice that the desired property (3) is the "Carleson embedding"

$$
\sum_{I \in \mathcal{D}}\left(\frac{1}{|I|} \int_{I} f d x\right)^{2} w_{I} \leq B\|f\|_{2}^{2}
$$


where $w_{I}=|I|^{2}, I \in \mathcal{D}$. The necessary and sufficient condition for such an embedding is (see [NTV1999], [NT1996])

$$
\sup _{J \in \mathcal{D}} \frac{1}{|J|} \sum_{I \subset J, I \in \mathcal{D}} w_{I}<\infty
$$

which is obviously fulfilled for $w_{I}=|I|^{2}, I \in \mathcal{D}$.

4.3. Part II of the Theorem 1.2. Take $\Omega=(0,2)$, and let $\left(v_{n}\right)_{n \geq 1}$ be the sequence in $L_{\mathbb{R}}^{2}((0,1), d x)$ constructed in Part I. Without loss of generality, we suppose that $B<1$. Then, the linear mapping $T: l^{2} \longrightarrow L^{2}(0,1)$ defined by $T \delta_{n}=v_{n}, n \geq 1\left(\delta_{n}\right.$ stands for the natural basis of $\left.l^{2}\right)$ is a (strict) contraction. Let $D_{T}=\left(I-T^{*} T\right)^{1 / 2}: l^{2} \longrightarrow l^{2}$ its defect operator, and $V: l^{2} \longrightarrow L^{2}(1,2)$ an arbitrary isometric operator. We naturally consider $L^{2}(0,2)$ as an orthogonal sum $L^{2}(0,2)=L^{2}(0,1) \oplus L^{2}(1,2)$ and set $U x=T x \oplus V D_{T} x$ for $x \in l^{2}$. Then, $U$ is isometric, $\|U x\|^{2}=\|T x\|^{2}+\left\|D_{T} x\right\|^{2}=\|x\|^{2}$, and hence $u_{2 n}:=U \delta_{n}$, $n=1,2, \ldots$ is an orthonormal basis in $F:=U l^{2} \subset L^{2}(0,2)$. Choosing an arbitrary orthonormal basis $\left(u_{2 n+1}\right)_{n>1}$ in the orthogonal complement $F^{\perp}$, we obtain an orthonormal basis $\left(u_{k}\right)_{k \geq 1}$ in $L^{2}(0,2)$ satisfying all requirements of the theorem (with $E=(0,1)$ ).

\subsection{A lapse of equidistribution between $u_{k}^{ \pm}(x)$.}

Proof. One can reordering the basis from 4.2 in order to get the following: there exists an orthonormal basis $\left(U_{k}\right)$ in $L_{\mathbb{R}}^{2}(0,2)$ such that

$$
\sum_{k=1}^{n}\left(U_{k}^{-}(x)\right)^{2}=o\left(\sum_{k=1}^{n}\left(U_{k}^{+}(x)\right)^{2}\right) \text { as } n \longrightarrow \infty x \in(0,1) \text {. }
$$

Indeed, it suffices to set

$$
\left(U_{k}\right): u_{2}, u_{4}, \ldots, u_{2 N_{1}}, u_{1}, u_{2 N_{1}+2}, \ldots, u_{2 N_{2}}, u_{3}, \ldots
$$

where the integers $N_{1}<N_{2}<\ldots$ increase sufficiently fast.

4.5. A minimal sequence can be positive. Let $u_{k}(x)=\frac{1}{1+\sqrt{2}}(1+\operatorname{Cos}(\pi k x)), k=$ $1,2, \ldots$ in $L_{\mathbb{R}}^{2}(0,1)$. Then $\left(u_{k}\right)$ spans $L_{\mathbb{R}}^{2}(0,1)$, is normalized and uniformly minimal (with the dual $\left.u_{k}^{\prime}=(2+\sqrt{2}) \operatorname{Cos}(\pi k x)\right)$, and $u_{k}(x) \geq 0$. In fact, the Fourier series with respect to $\left(u_{k}\right)$ of a function $f \in L_{\mathbb{R}}^{2}(0,1), \sum_{k>1}\left(f, u_{k}^{\prime}\right) u_{k}$, converges to $f$, if $f$ is (for example) Dini continuous at $x=0$ and $f(0)=0$. However, $\left(u_{k}\right)$ is not a basis.

The question of the existence of non-negative Schauder basis in $L^{p}, p>1$ is open to the best of our knowledge. Detailed discussion can be found in [PS2016]. For $p=1$, as it is already mentioned, non-negative Schauder basis exists, see [JS2015].

\section{REFERENCES}

[Aru1966] F.G. Arutyunyan, Distribution of positive and negative values of functions forming an unconditional basis in the space $L_{p}(0,1)$ (Russian. Armenian and English summaries), Izv. Akad. Nauk Armyan. SSR, Ser. Mat. 1:1 (1966), 79-87.

[Ber1968] Ju. M. Berezanskii, Expansions in Eigenfunctions of Selfadjoint Operators, Transl. Math. Monographs, vol.17, Amer. Math. Soc., Providence, RI, 1968.

[Gle2016] V. D. Glezer, Vision and Mind. Modeling mental functions. Psychology Press, N.J., 2016 (first edition 1985, 1995).

[JS2015] W. B. Johnson, G. Schechtman, A Schauder basis for $L_{1}(0, \infty)$ consisting of non-negative functions. Illinois Journal of Mathematics Volume 59, Number 2, Summer 2015, Pages 337-344.

[Koz1948] V. Ya. Kozlov, On the distribution of positive and negative values of complete orthonormal function systems, Matem. Sbornik, t.23(65),1948, $\mathrm{N}^{\circ} 3,475-480$ (in Russian). 
[KPS1978] S. G. Krein, Yu. I. Petunin, E. M. Semenov, Interpolation of linear operators (Russian), Moscow, "Nauka", 1978 (English transl.: American Math. Soc., Transl. Math. Monogr. vol 54, Providence, R.I., 1982).

[MSS2015] A. Marcus, D. Spielman, and N. Srivastava, Interlacing families II: Mixed characteristic polynomials and the Kadison-Singer problem, Ann. of Math. (2) 182(2015), no.1, 327-350.

[MaN1983] A. Máté and P. Nevai, Orthogonal polynomials and absolutely continuous measuresin C .K .Chui et al., eds., Approximation Theory IV, vol.103, Academic Press, N.Y., 611-617.

[NT1996] F. Nazarov and S. Treil, The hunt for Bellman function: applications to estimates of singular integral operators and to other classical problems in harmonic analysis. (Russian), Algebra i Analyz, 8:5 (1996), 32-162; English Transl. in St.Petersburg Math. J., 8:5 (1997), 721-824.

[NTV1999] F. Nazarov, S. Treil, and A .Volberg, The Bellman functions and two-weight inequalities for Haar multipliers, J. Amer. Math. Soc., 12:4 (1999) 909-928.

[Ovs1980] R. I. Ovsepyan, Extraction of lacunary subsystems from general systems with a property of absolute convergence, (Russian), Mat. Sbornik 111(153):4 (1980), 522-531, 623.

[Ped2002] S.Pedersen, Absolutely continuous Jacobi operators , Proc. Amer. Math. Soc., 130:8 (2002), 2369-2376.

[PS2016] A. M. Powell, A. H. Spaeth, Nonnegativity constraints for structured complete systems, Trans. AMS, Volume 368, Number 8, August 2016, Pages 5783-5806 http://dx.doi.org/10.1090/tran/6562

[Sim2005] B. Simon, Orthogonal polynomials on the unit circle, Part 1: classical theory, Coll. Publ. 54:1, Amer. Math. Soc., Providence, 2005.

[Sz1975] G. Szegö, Orthogonal polynomials, 4th edition, AMS Colloq. Publ., vol.23, Amer Math Soc, Providence, RI, 1975.

[Szw2003] R. Szwarc, Absolute continuity of certain unbounded Jacobi matrices, in M.D.Buhmann and D.H.Mache, eds., Advanced Problems in Constructive Approximation, International Series in Numerical Mathematics, Birkhaüser, Basel, vol.12, 255-262.

[Woj1996] P. Wojtaszczyk, Banach spaces for analysts, Cambridge Univ. Press, 1996.

Institut de Mathématiques de Bordeaux, Université de Bordeaux, Talence, France, And - Chebyshev Laboratory, StPetersburg University

E-mail address: nikolski@math.u-bordeaux.fr (N. Nikolski)

Department of Mathematics, Michigan State University, East Lansing, Mi 48823, USA

E-mail address: volberg@math.msu.edu (A. Volberg) 